Sir,

\section{Multiple sclerosis presenting with debilitating \\ Uhthoff's symptom}

Uhthoff's symptom, the precipitation by or worsening of symptoms with activities associated with a rise in body temperature, is a well-recognised phenomenon, classically occurring in patients with established multiple sclerosis (MS) and a history of optic neuritis. ${ }^{1,2} \mathrm{We}$ present a case of probable MS presenting with a long history of worsening intermittent blurring of vision exacerbated by exercise or elevated temperature, in the absence of any previous neurological symptoms. The effect of exercise on the patient's visual function had become marked and debilitating.

\section{Case report}

A 29-year-old man presented with a 14-year history of intermittent bilateral blurring of vision. This was initially precipitated by exercise and hot baths, but in the months preceding presentation the attacks had become more severe and frequent, and could even occur spontaneously. The duration of each episode had also increased with time, and episodes were lasting up to several hours at the time of presentation. His symptoms were interfering greatly with daily activities. There were no symptoms suggestive of a previous attack of optic neuritis and there was no history of other neurological symptoms. He had previously been fit and well, and apart from a 6-month course of isotretinoin for acne at the age of 15 years had been on no medications.

On examination at rest, visual acuity was $6 / 9$ in each eye. He identified 14 of 17 Ishihara plates with his left eye but only the test plate with the right. There was a mild right relative afferent pupillary defect, and bilateral temporal optic disc pallor. There was gaze-evoked nystagmus in dextroversion. Examination by a neurologist revealed equivocal upgoing plantar responses but no other abnormalities.

After 5 minutes' brisk walking up and down stairs, his visual acuities fell to 1/60 right and 3/60 left. There was also a profound effect of exercise on his visual fields (Fig. 1). It can be seen from Fig. 1, which shows the Humphrey 24-2 field of the right eye before and after the same period of exercise, that sensitivity decreased dramatically as a result of exertion. The picture in the left eye was very similar. The mean deviation in the right eye before exercise was $-12.52 \mathrm{~dB}$ and that in the left, -11.92 $\mathrm{dB}$. After exercise these values were $-21.05 \mathrm{~dB}$ and $-20.20 \mathrm{~dB}$ respectively.

A series of investigations were performed. Positive findings were the presence of oligoclonal bands in the cerebrospinal fluid, severely abnormal visual and auditory evoked responses, and MRI evidence of lesions in the brainstem, optic nerves and extensively in the periventricular white matter (Fig. 2).
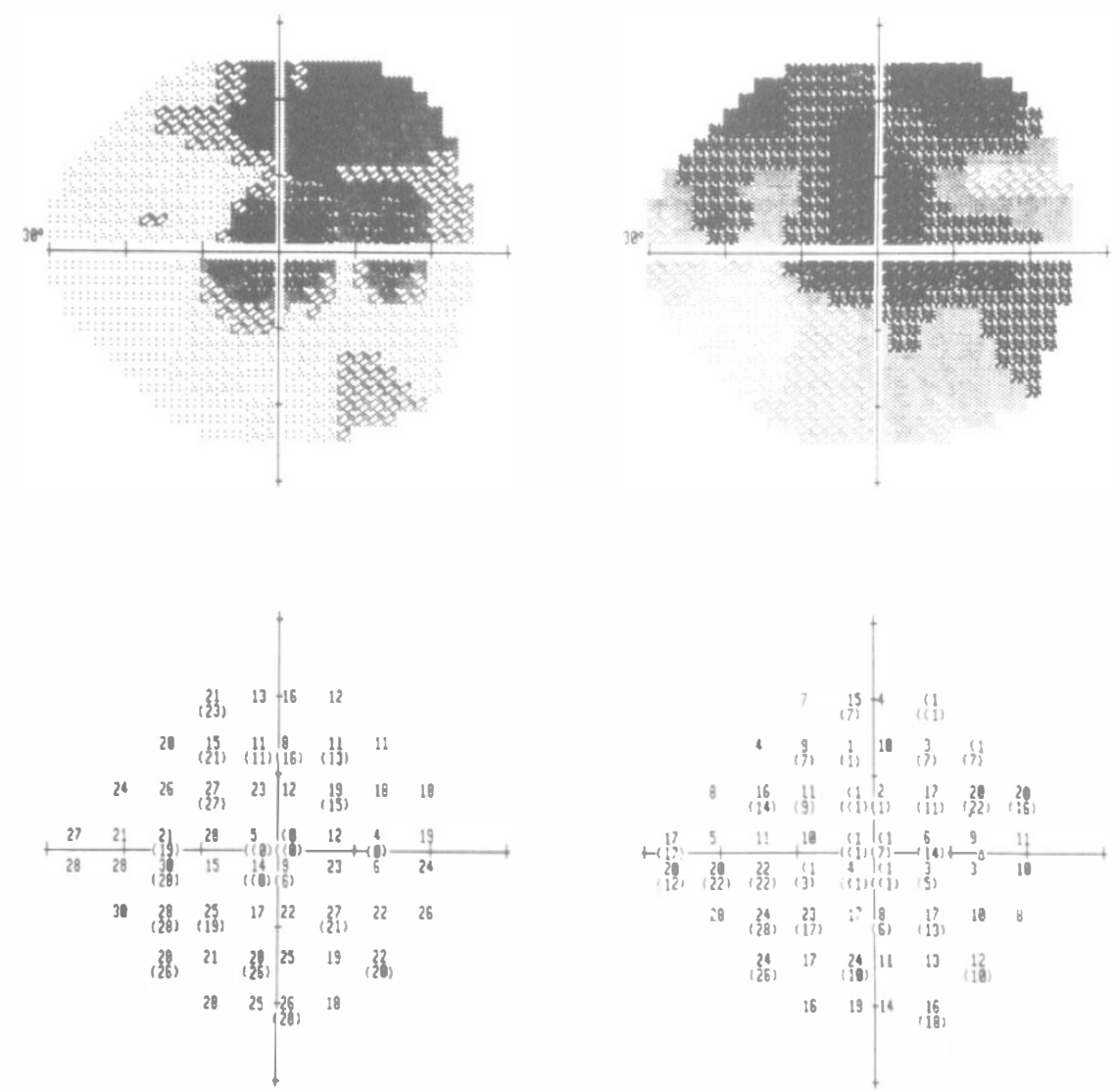

Fig. 1. Humphrey 24-2 full threshold field of the right eye, showing grey scales (top) and sensitivity (bottom). The left-hand plots show the field at rest, and those on the right the fields after 5 minutes' exercise. 


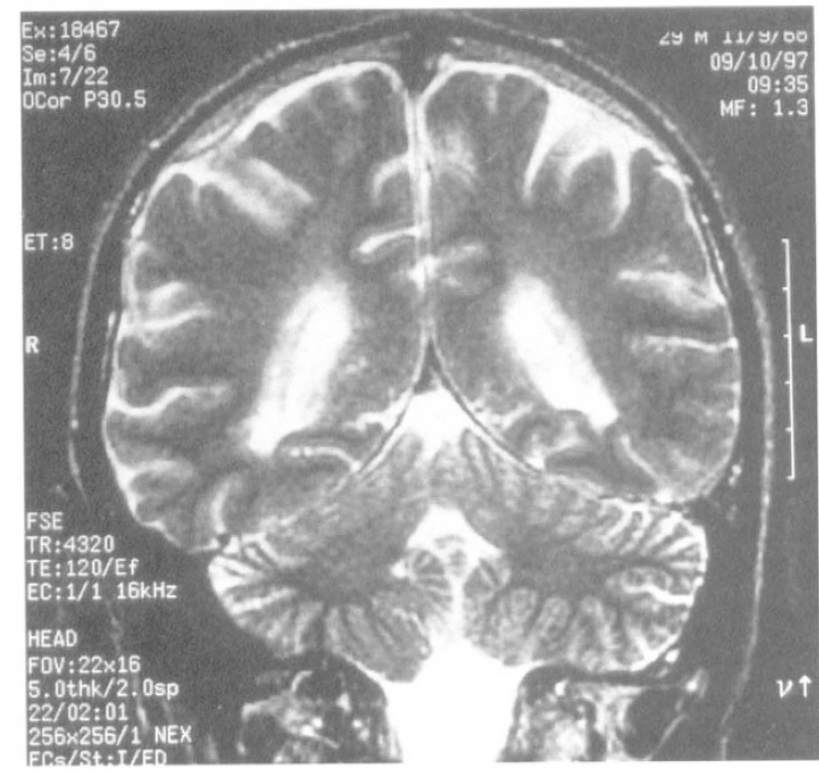

Fig. 2. Coronal T2-weighted MRI scan of the brain demonstrating extensive plaques in the periventricular white matter.

Due to the severity of his complaints and his inability to work, the patient was treated with three pulses of intravenous methylprednisolone. Since then his symptoms and visual fields have gradually improved. On examination 1 month after treatment, at rest, the mean deviation of the field in the right eye before exercise was $-4.74 \mathrm{~dB}$, and in the left $-4.75 \mathrm{~dB}$, falling after 5 minutes' exercise to -12.58 and $-10.55 \mathrm{~dB}$ respectively.

\section{Comment}

Since Uhthoff's original paper in $1889,{ }^{1}$ his symptom has received much attention in the literature. It has been reported to occur in up to half of patients with optic neuritis, ${ }^{3}$ and is generally believed to be a direct effect of temperature on conduction in severely demyelinated axons. ${ }^{4}$ The symptom has been said to be associated with an increased incidence of recurrent optic neuritis and also of MS. ${ }^{3}$ It is also more rarely found in other neurological conditions. ${ }^{5}$

Progressive (non-variable) visual failure is an uncommon but recognised presentation of MS. ${ }^{6}$ Reports of MS presenting with intermittent blurring are also very unusual; $^{2}$ to our knowledge, however, this is the first reported case where Uhthoff's symptom has occurred as the patient's only problem in such a progressive and debilitating manner. The worsening with time of our patient's symptoms suggests progressive MS (as opposed to relapsing disease); also the occurrence of Uhthoff's symptom has been found to be associated with more extensive demyelination. ${ }^{2}$ Progressive disease generally carries a worse prognosis, although in MS sufferers with predominantly sensory disturbance the outlook can be somewhat brighter. ${ }^{7}$ Whilst it is possible that the use of high-dose systemic steroids did have some effect on our patient's symptoms, this must be seen in the light of previous evidence that steroids have no influence on the duration or frequency of Uhthoff's phenomenon. ${ }^{3}$ Consequently we cannot advocate their routine use in this setting.

\section{References}

1. Uhthoff $\mathrm{W}$. Untersuchungen über die bei der multiplen Herdsklerose vorkommenden Augenstorungen. Arch Psychiat Nervenker 1889;21:55-116:303-410.

2. Persson HE, Sachs C. Visual evoked potentials elicited by pattern reversal during provoked visual impairment in multiple sclerosis. Brain 1981;104:369-82.

3. Scholl GB, Song H-S, Wray SH. Uhthoff's symptom in optic neuritis: relationship to magnetic resonance imaging and development of multiple sclerosis. Ann Neurol 1991;30:180-4.

4. Rasminsky M. The effects of temperature on conduction in demyelinated single nerve fibers. Arch Neurol 1973;28:287-92.

5. Perkin GD, Rose FC. Uhthoff's syndrome. In: Perkin GD, Rose FC, editors. Optic neuritis and its differential diagnosis. Oxford: Oxford University Press, 1979:183-93.

6. Ormerod IEC, McDonald WI. Multiple sclerosis presenting with progressive visual failure. J Neurol Neurosurg Psychiatry 1984;47:943-6.

7. Compston DAS. Demyelinating disorders of the central nervous system. In: Weatherall DJ, Ledingham JGG, Warrell DA, editors. Oxford textbook of medicine. 3rd ed. Oxford: Oxford University Press, 1996:3989-98.

Alan W.D. Fitt

Michael A. Burdon

Birmingham \& Midland Eye Centre

Birmingham, UK

Mr Alan W.D. Fitt

Birmingham \& Midland Eye Centre

City Hospital NHS Trust

Dudley Road

Birmingham B18 7QU, UK

Tel: +44 (0)121 5543801

Fax: +44 (0)121507 6853

e-mail: alfitt@globalnet.co.uk

Sir,

Congenital oculomotor palsy associated with brainstem hypoplasia

Oculomotor nerve palsies present at birth are usually thought to be secondary to maldevelopment, intrauterine injury or birth trauma. The association of congenital third nerve palsy with systemic and neurological

abnormalities is variously reported in the literature. ${ }^{1-5}$ In Miller's series, ${ }^{1}$ none of the 13 congenital cases had physical or neurological abnormalities. In the series of Balkan and Hoyt, ${ }^{2}$ however, and in the more recent investigations of Hamed $^{3}$ and Tsalousmas ${ }^{4}$ using modern neuroimaging studies, accompanying neurological or developmental defects are reported to be more frequent and widespread than was once thought. Some cases of congenital oculomotor palsy may therefore be caused by central lesions. We report an 8-month-old female infant with congenital oculomotor nuclear palsy. A magnetic resonance imaging (MRI) scan demonstrated right side brainstem hypoplasia associated with hypoplasia of the corpus callosum. 